\section{Cureus}

Received 05/16/2017

Review began 05/24/2017

Review ended 03/28/2018

Published 04/03/2018

\section{(c) Copyright 2018}

Thawani et al. This is an open access article distributed under the terms of the Creative Commons Attribution License CC-BY 3.0., which permits unrestricted use, distribution, and reproduction in any medium, provided the original author and source are credited.

\title{
Tracheomediastinal Fistula: Rare Complication of Treatment with Bevacizumab
}

\author{
Rajat Thawani ${ }^{1}$, Anna Thomas ${ }^{2}$, Kshitij Thakur ${ }^{3}$ \\ 1. Internal Medicine Resident, Maimonides Medical Center, Brooklyn, USA 2. Internal Medicine, Crozer- \\ Chester Medical Center, Upland, USA 3. Internal Medicine, University of Kentucky School of Medicine, \\ Lexington, USA
}

$\square$ Corresponding author: Rajat Thawani, rajat13@gmail.com

Disclosures can be found in Additional Information at the end of the article

\section{Abstract}

Tracheomediastinal fistula is a rare condition caused by multiple etiologies. We present a case of a patient of lung carcinoma receiving chemotherapy. A 63-year-old woman presented to the emergency room with a two-month history of worsening cough and shortness of breath. She was being treated with pemetrexed and bevacizumab for Stage IV non-small cell lung carcinoma. Chest X-ray showed a mass in the lung with mediastinal adenopathy. Computed tomography (CT) scan showed a perforation, confirmed with bronchoscopy. She had a secondary infection and she was started on intravenous antibiotics. The patient decided to continue care in a hospice. We present a rare complication of bevacizumab which has been only reported once in literature. Bevacizumab is known to cause tracheal fistulas when coupled with like invasive procedures. In our case, the patient developed a fistula without any invasive interventions. We advise that physicians using bevacizumab should be aware of the possibility of having such fistulas.

Categories: Oncology, Pulmonology

Keywords: bevacizumab, tracheomediastinal fistula, tracheal fistula, non-small cell lung cancer

\section{Introduction}

Tracheomediastinal fistula is a rare condition usually with a fatal outcome [1]. A literature review done showed that the listed causes of this fistula include tracheal or mediastinal lymphoma [1], radiation/laser therapy [2,3], chemotherapy in patients with lung carcinomas, notably bevacizumab [4], and surgical interventions [5]. We present a case where a patient receiving chemotherapy with bevacizumab came to the emergency room (ER) with shortness of breath and worsening cough.

\section{Case Presentation}

A 63-year-old female presented to the emergency room with a two-month history of worsening cough and shortness of breath. Over the course of this time, her symptoms were worsening and her cough became non-productive. She was using $2 \mathrm{~L}$ oxygen for her symptoms. Her past medical history included a history of Stage IV non-small cell lung carcinoma (adenocarcinoma) with adrenal metastasis. She received first-line chemotherapy with pemetrexed and carboplatin for three months to which she did not respond, after which she was switched to pemetrexed with biweekly bevacizumab. Her last chemotherapy was two weeks before presentation. 


\section{Cureus}

Significant vitals at presentation were the temperature of $99.6^{\circ} \mathrm{F}$, heart rate 102 , respiratory rate 19 , pulse oximetry $95 \%$ on $4 \mathrm{~L}$ oxygen and blood pressure of 141/75 $\mathrm{mm} \mathrm{Hg}$. Examination showed bilateral equal and adequate air entry in lungs. Initial chest X-ray demonstrated a $2.5 \mathrm{x}$ $4.5 \times 3.9 \mathrm{~cm}$ upper lobe mass with mediastinal adenopathy and enlarged pulmonary arteries.

Blood investigations showed lymphocytosis (white blood cells $14.2 \times 10^{9} / \mathrm{L}$ ). A computed tomography (CT) of her chest showed a possible tracheal invasion of the tumor with perforation (Figure 1). She was admitted and scheduled for bronchoscopy, which revealed a right-sided tracheomediastinal fistula (TMF) proximal to the carina (Figure 2). Blood cultures grew Streptococcus pneumonie and Klebsiella pneumonie. Thoracic surgery consult was obtained but surgery was deferred until her infection resolved. She was started on intravenous Vancomycin and Piperacillin-Tazobactam, and received them for 42 days with close follow-up. The patient wished against undergoing surgery later and decided to continue care in a hospice.

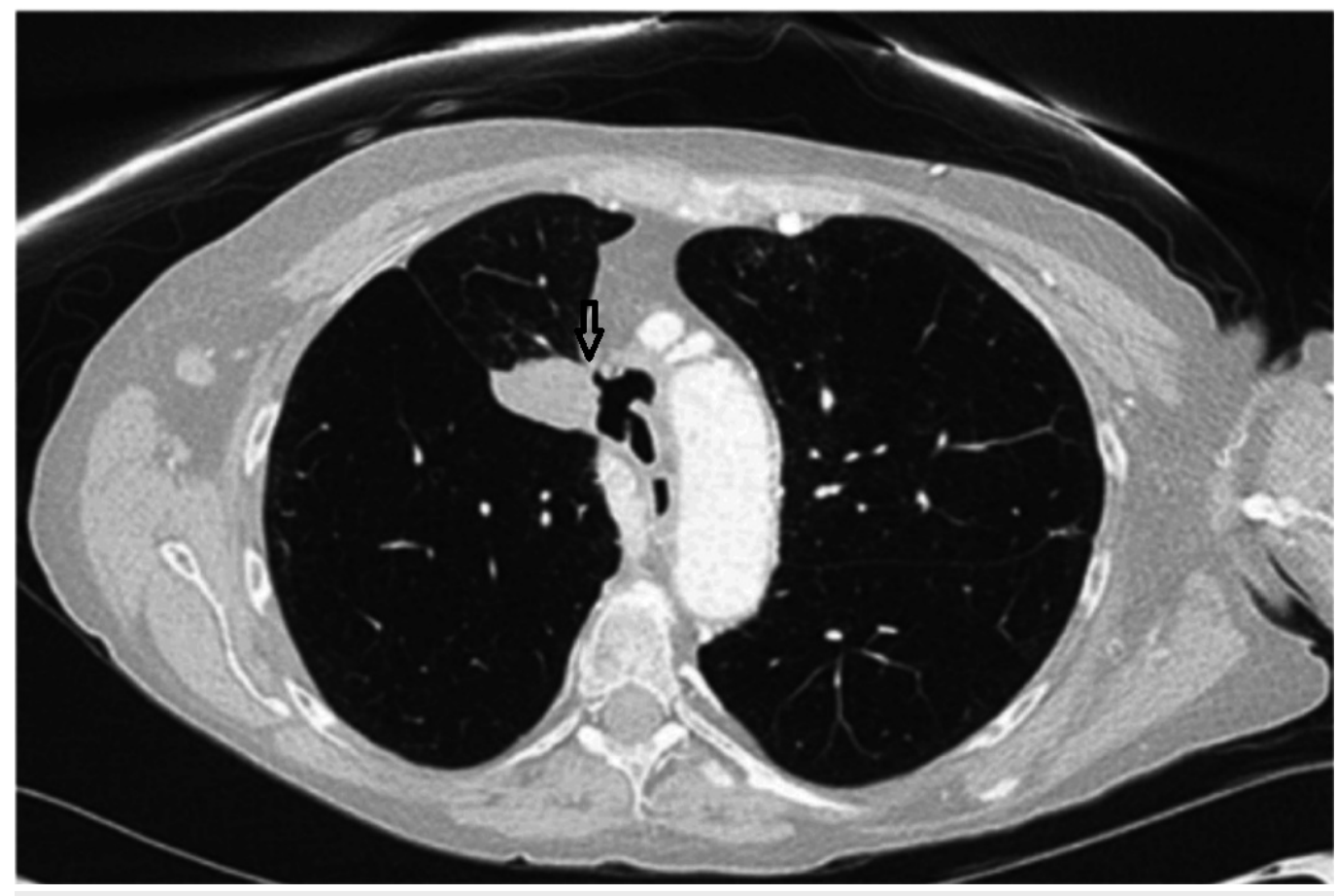

FIGURE 1: Computed tomography (CT) scan showing tumor near the mediastinum and formation of a fistula. 


\section{Cureus}
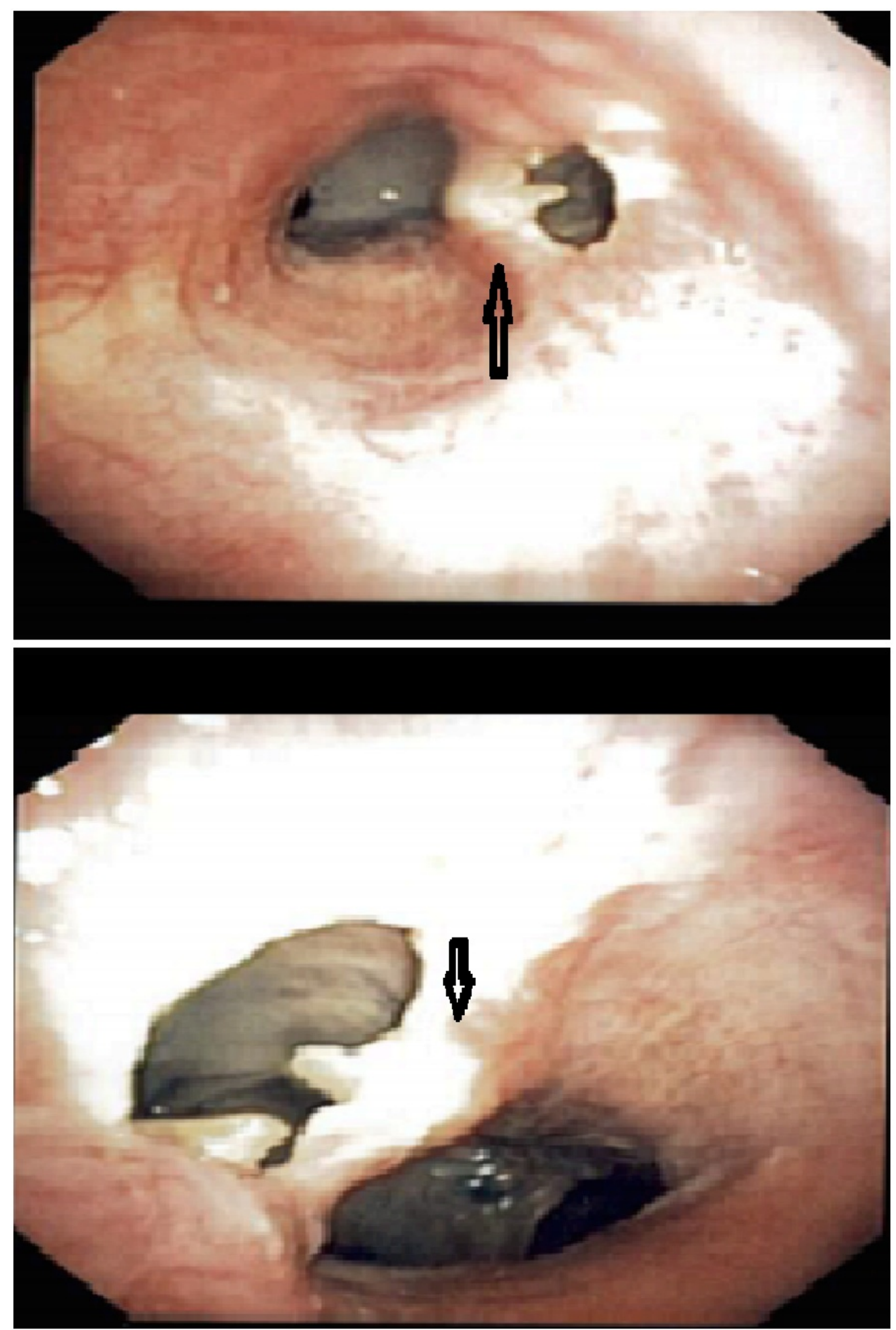

FIGURE 2: Bronchoscopy images showing the fistula.

Arrow pointing at the fistula. 


\section{Discussion}

Bevacizumab has been shown to improve outcomes in patients with non-small cell lung cancer (NSCLC), apart from other malignancies like colorectal carcinoma, renal cell carcinoma, glioblastoma multiforme and gynecologic malignancies. At the same time, it has been associated with significant side effects like diarrhea, arterial and venous thromboembolism, hemorrhage and gastrointestinal perforations [6]. In patients of NSCLC, tracheoesophageal fistula (TEF) has been reported when bevacizumab is combined with radiotherapy [7]. There are multiple hypotheses about the mechanism of the causes of fistula. In NSCLC, the antiangiogenic action of the drug in combination with esophageal or tracheal injury has been postulated as a cause of TEF. It is now a recognized adverse effect of patients who have received bevacizumab along with a multi-modality treatment of lung cancers.

On the other hand, TMF is an even rarer of bevacizumab reported only once by McCarthy and Hamel where a patient suffering from NSCLC received chemotherapy and radiation therapy [4]. But our patient never received any local treatment for the lung cancer, or never underwent any instrumentation and went on to develop TMF secondary to only bevacizumab.

There is no consensus on the treatment of this condition and is individualized. It may vary from surgical or endoscopic closure [2] to plasma coagulation [3], fibrin glue [5], or even autologous adipose-derived stem cells [2]. We treated our patient's secondary infections before proceeding with definitive surgery as it is recommended in TEF [8-10].

The presentation of TMF in McCarthy and Hamel and our case was non-specific, and may not be detected by routine chest X-rays. We hypothesized that the TMF was formed secondary to treatment because there was no evidence of progression of the disease, and the patient was on treatment during the episode. Hence, we advise that physicians using bevacizumab should be aware of the possibility of having such rare fistulas and should consider it as an important differential in patients being treated with it. Any common or non-specific symptoms may be a sign of these rare fistulas and a thorough workup is warranted. Additionally, our case also shows that bevacizumab can cause these symptoms even in the absence of local therapy (radiation or surgery), unlike what has been published in the previous literature.

\section{Conclusions}

Tracheomediastinal fistula could be a potentially lethal complication in patients being treated with bevacizumab. Physicians treating patients with this should be aware of this complication, and if symptoms suggest, terminate therapy early and take measures to treat the condition.

\section{Additional Information Disclosures}

Human subjects: Consent was obtained by all participants in this study. Conflicts of interest: In compliance with the ICMJE uniform disclosure form, all authors declare the following: Payment/services info: All authors have declared that no financial support was received from any organization for the submitted work. Financial relationships: All authors have declared that they have no financial relationships at present or within the previous three years with any organizations that might have an interest in the submitted work. Other relationships: All authors have declared that there are no other relationships or activities that could appear to have influenced the submitted work.

\section{References}

1. Choudhary C, Gildea TR, Salman R, Guzman ED, Mehta AC: Management of 
tracheomediastinal fistula using self-expanding metallic stents. Ann Thorac Surg. 2008, 85:1800-1802. 10.1016/j.athoracsur.2007.11.045

2. Alvarez PD-A, García-Arranz M, Georgiev-Hristov T, García-Olmo D: A new bronchoscopic treatment of tracheomediastinal fistula using autologous adipose-derived stem cells. Thorax. 2008, 63:374-376. 10.1136/thx.2007.083857

3. Ucer M, Ordu C, Pilanc KN, Dalar L: Tracheomediastinal fistula in a patient with lung adenocarcinoma and its treatment with argon plasma coagulation: a case report. Medicine (Baltimore). 2014, 93:e156. 10.1097/MD.0000000000000156

4. McCarthy J, Hamel J: Tracheal-mediastinal fistula post-chemoradiation therapy. West J Emerg Med. 2014, 15:876-877. 10.5811/westjem.2014.8.23438

5. Weiss G, Wex C, Lippert H, Schreiber J, Meyer F: Successful endoscopic treatment of a postoperative tracheomediastinal fistula caused by anastomotic insufficiency after esophageal resection with fibrin glue. Pol Przegl Chir. 2015, 86:537-539. 10.2478/pjs-2014-0095

6. Hompes D, Ruers T: Review: incidence and clinical significance of Bevacizumab-related nonsurgical and surgical serious adverse events in metastatic colorectal cancer. Eur J Surg Oncol. 2011, 37:737-746. 10.1016/j.ejso.2011.06.004

7. Spigel DR, Hainsworth JD, Yardley DA, et al.: Tracheoesophageal fistula formation in patients with lung cancer treated with chemoradiation and bevacizumab. J Clin Oncol. 2010, 28:43-48. 10.1200/JCO.2009.24.7353

8. Robins B, Das AK: Anesthetic management of acquired tracheoesophageal fistula: a brief report. Anesth Analg. 2001, 93:903-905. 10.1097/00000539-200110000-00020

9. Chauhan SS, Long JD: Management of tracheoesophageal fistulas in adults . Curr Treat Options Gastroenterol. 2004, 7:31-40. 10.1007/s11938-004-0023-3

10. Shah CP, Yeolekar ME, Pardiwala FK: Acquired tracheo-oesophageal fistula. J Postgrad Med. 1994, 40:83-84. 